\title{
The Composition of Linear Differential Systems
}

By J. M. Whitraker, Pembroke College, Cambridge.

(Received 7th May, 1931. Read 6th June, 1931.)

\section{§1. Introduction.}

A common method of solving a linear differential equation consists in expressing the differential operator as a product of factors. The possibility of doing so has been studied extensively by Vessiot ${ }^{1}$, following the work of Picard and Drach, on the lines of the Galois theory of algebraic equations. The analogous process of resolving a linear differential system, consisting of an equation together with boundary conditions, into two or more systems of lower order does not seem to have been investigated. Such a resolution is not always possible, even in cases where the differential equation can be factorised. Thus the system

$$
\left\{\begin{array}{l}
\frac{d^{2} y}{d x^{2}}=f(x) \\
y(0)=\alpha, \quad y^{\prime}(1)=\beta
\end{array}\right.
$$

is equivalent to the systems

but the system

$$
\left\{\begin{array} { l } 
{ \frac { d z } { d x } = f ( x ) , } \\
{ z ( 1 ) = \beta }
\end{array} \quad \left\{\begin{array}{l}
\frac{d y}{d x}=z(x) \\
y(0)=\alpha
\end{array}\right.\right.
$$

$$
\left\{\begin{array}{l}
\frac{d^{2} y}{d x^{2}}=f(x) \\
y(0)=a, \quad y(1)=\gamma
\end{array}\right.
$$

cannot be so resolved.

The present paper is a contribution to this problem. The product of two operator systems is defined and it is shown that the Green's function of the product system is the product (by composition of the second kind) of the Green's functions of the component systems. The two products of a system with the adjoint system are shown to be self-adjoint, and their eigenfunctions to be useful in solving the original system.

I See the account in Picard's Traité d'Analyse, 3 (1928), chap. 17. 
Ordinary differential systems only are discussed, but it will be evident that the definition of the product of two systems can be extended to partial differential systems.

\section{§2. Resolution of a differential system.}

Let

$$
\begin{aligned}
& P=\frac{d^{p}}{d x^{p}}+p_{1}(x) \frac{d^{p-1}}{d x^{p-1}}+\ldots+p_{p}(x) \\
& Q=\frac{d^{q}}{d x^{q}}+q_{1}(x) \frac{d^{q-1}}{d x^{q-1}}+\ldots+q_{q}(x)
\end{aligned}
$$

be two differential operators. It is assumed that $p_{1}(x), \ldots, p_{p}(x)$ are continuous in $(a, b)$; and that $q_{1}(x), \ldots, q_{q}(x)$, together with their first $p$ derivatives, are continuous in $(a, b)$. Consider the system

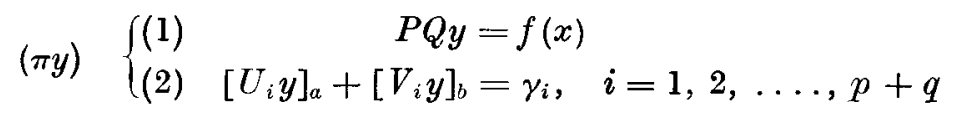

where $U_{i}, V_{i}$ are differential operators of orders $\leqslant p+q-1$.

If there exist $p$ linearly independent combinations of (2) of the form

$$
\text { (3) }\left[A_{i} Q y\right]_{a}+\left[B_{i} Q y\right]_{b}=\delta_{i}, \quad i=1,2, \ldots, p
$$

where $A_{i}, B_{i}$ are differential operators of order $\leqslant p-1,(\pi y)$ can be resolved as follows. Write

$$
Q y=z
$$

so that (1), (3) give

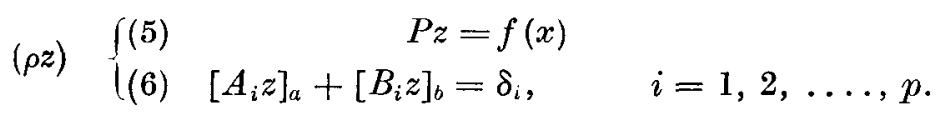

There are $q$ linear combinations of (2) which, together with (3). form a linearly independent set. In general these will involve derivatives of orders up to $p+q-1$. By use of (4), and the equations obtained by differentiating it, the derivatives of order $\geqslant q$ can be eliminated. In this way $q$ linearly independent boundary conditions are obtained,

$$
\text { (7) }\left[C_{i} y\right]_{a}+\left[D_{i} y\right]_{b}=\epsilon_{i}, \quad i=1,2, \ldots, q
$$

the constants $\epsilon_{i}$ depending on $z(a), z(b), z^{\prime}(a), \ldots, z^{(p-1)}(b) . \quad(\pi y)$ is then equivalent to $(\rho z)$ combined with the system $(\sigma y)$ consisting of (4) and (7). In $(\sigma y), z(x)$ is regarded as a known function, having been determined by $(\rho z)$. 
Thus the system

$$
\left\{\begin{array}{c}
\frac{d^{2} y}{d x^{2}}+\left(x-e^{x}\right) \frac{d y}{d x}+\left(1-x e^{x}\right) y=f(x) \\
y^{\prime}(0)+y(1)=\gamma_{1}, \quad y^{\prime}(1)=\gamma_{2}
\end{array}\right.
$$

is equivalent to the systems

$$
\left\{\begin{array} { l } 
{ \frac { d z } { d x } - e ^ { x } z = f ( x ) } \\
{ z ( 0 ) + z ( 1 ) = \gamma _ { 1 } + \gamma _ { 2 } }
\end{array} \quad \text { and } \quad \left\{\begin{array}{rl}
\frac{d y}{d x}+x y=z(x) \\
y(1)=z(1)-\gamma_{2}
\end{array}\right.\right.
$$

§3. The product of two operator systems.

The foregoing work suggests a definition of the product of two operator systems.

$A$ system

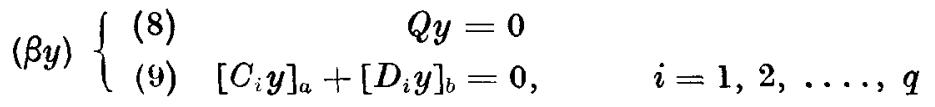

being given, the system obtained by multiplying it by the operator system

$$
\text { (a) }\left\{\begin{array}{cc}
(10) & P \\
(11) & {\left[A_{i}\right]_{a}+\left[B_{i}\right]_{b}}
\end{array} \quad i=1,2, \ldots, p\right.
$$

is the system

$$
(\beta a y)\left\{\begin{array}{ccc}
(12) & P Q y=0 \\
(13) & {\left[A_{i} Q y\right]_{a}+\left[B_{i} Q y\right]_{b}=0,} & i=1,2, \ldots, p \\
(14) & {\left[C_{i} y\right]_{a}+\left[D_{i} y\right]_{b}=0,} & i=1,2, \ldots, q .
\end{array}\right.
$$

The notation $(\beta a y)$, rather than $(\alpha \beta y)$, is suggested by the order of the Green's functions in the integral of Theorem 1.

Now assume that $(\alpha y),(\beta y)$, are incompatible, so that their Green's functions $K(x, \zeta), L(x, \zeta)$ exist and are unique. $K(x, \zeta)$ has the properties ${ }^{1}$

(i) it is continuous and possesses continuous derivatives of orders up to and including $p-2$, when $a \leqslant x \leqslant b$.

(ii) its derivative of order $p-1$ is discontinuous at a point $\zeta$ in $(a, b)$, having an upward jump of amount unity.

(iii) it satisfies the system $(a y)$ at all points of $(a, b)$ except $\zeta$.

It is found that the Green's function of $(\beta a y)$ is the product by composition of the second kind ${ }^{2}$ of those of $(\beta y),(\alpha y)$.

3 Cf. Ince, Ordinary Differential Equations (1927), 254.

2 Cf. Volterra, Theory of Functionals (1930), 99. 
Theorem 1. The Green's function of $(\beta a y)$ is

$$
\int_{a}^{b} L(x, \eta) K(\eta, \zeta) d \eta
$$

when $K(x, \zeta)$ is the Green's function of $(\alpha y)$ and $L(x, \zeta)$ that of $(\beta y)$.

Denote the integral by $M(x, \zeta)$. Then, using the properties of $L(x, \zeta)$ corresponding to (i), (ii), (iii),

so that

$$
\begin{aligned}
& \frac{\partial^{s} M}{\partial x^{s}}=\int_{a}^{b} \frac{\partial^{s} L}{\partial x^{s}} K(\eta, \zeta) d \eta, \quad s=1,2, \ldots, q-1 \\
& \frac{\partial^{q} M}{\partial x^{q}}=\int_{a}^{b} \frac{\partial^{q} L}{\partial x^{q}} K(\eta, \zeta) d \eta+K(x, \zeta)
\end{aligned}
$$

$$
\begin{aligned}
Q M(x, \zeta) & =\int_{a}^{b}\{Q L(x, \eta)\} K(\eta, \zeta) d \eta+K(x, \zeta) \\
& =K(x, \zeta)
\end{aligned}
$$

since $L(x, \zeta)$ satisfies (8). Again, (16) can be written

$$
\frac{\partial^{q} M}{\partial x^{q}}=\int_{a}^{b}\left\{-q_{1}(x) \frac{\partial^{q-1} L}{\partial x^{q-1}}-\ldots-q_{q}(x) L\right\} K(\eta, \zeta) d \eta+K(x, \zeta)
$$

so that

$$
\begin{aligned}
\frac{\partial^{q+1} M}{\partial x^{1+1}}=\int_{a}^{b}\left\{-q_{1}(x) \frac{\hat{\theta}^{q} L}{\partial x^{q}}-q_{1}{ }^{\prime}(x) \frac{\partial^{q-1} L}{\partial x^{q-1}}-\ldots\right\} K(\eta, \zeta) d \eta \\
\\
\quad-q_{1}(x) K(x, \zeta)+\frac{\partial K}{\partial x} \\
=\int_{a}^{b}\left\{q_{1}{ }^{2}(x) \frac{\partial^{q-1} L}{\partial x^{q-1}}+\ldots\right\} K(\eta, \zeta) d \eta-q_{1}(x) K(x, \zeta)+\frac{\partial K}{\partial x}
\end{aligned}
$$

and so on, the derivative of $L$ of order $q$ under the integral sign being always replaced by derivatives of lower order. In this way it is found that

$$
\frac{\partial^{p+q-1} M}{\partial x^{p+q-1}}=\text { a continuous function }+\frac{\partial^{p-1} K}{\partial x^{p-1}} .
$$

These equations show that $M$ and its first $p+q-2$ derivatives are continuous and that the derivative of order $p+q-1$ has an upward jump at $\zeta$ of amount unity. (17) shows that

$$
P Q M=P K=0
$$

and it is easy to see from (15), (17), that $M(x, \zeta)$ satisfies (13), (14). Thus $M(x, \zeta)$ must be the Green's function of $(\beta a y)$. 
It follows that the multiplication of systems is associative but in general not commutative.

§4. Adjoint systems.

It is known that the Green's function of the system $\left(\alpha^{\prime} y\right)$ adjoint to $(a y)$ is ${ }^{1}$

$$
(-)^{p} K(\zeta, x)
$$

It follows that if $(\alpha),(\beta), \ldots,(\kappa),(\lambda)$ are any operator systems and $\left(\alpha^{\prime}\right), \ldots,\left(\lambda^{\prime}\right)$ their adjoints, then

$$
(\alpha \beta \ldots \kappa \lambda)^{\prime}=\left(\lambda^{\prime} \kappa^{\prime} \ldots \beta^{\prime} \alpha^{\prime}\right)
$$

and in particular if $(\alpha)$ is any system, the systems $\left(\alpha \alpha^{\prime}\right),\left(\alpha^{\prime} \alpha\right)$ are selfadjoint. The Green's function of $\left(\alpha \alpha^{\prime} y\right)$ is

$$
(-)^{p} \int_{a}^{b} K(x, \eta) K(\zeta, \eta) d \eta=(-)^{p} \bar{K}(x, \zeta)
$$

in the notation of Schmidt2, while that of $\left(a^{\prime} \alpha y\right)$ is

$$
(-)^{p} \int_{a}^{b} K(\eta, x) K(\eta, \zeta) d \eta=(-)^{p} \underline{K}(x, \zeta)
$$

Let $\psi_{n}(x)$ be an eigenfunction ${ }^{3}$ of $K(x, \zeta)$ corresponding to an eigenvalue $\lambda_{n}^{2}$, so that

$$
\phi_{n}(x)=\lambda_{n} \int_{a}^{b} K(x, \zeta) \psi_{n}(\zeta) d \zeta
$$

is an eigenfunction of $\bar{K}(x, \zeta)$ for the same eigenvalue. $\phi_{n}(x), \psi_{n}(x)$ are thus eigenfunctions of the systems $\left(\alpha \alpha^{\prime} y\right),\left(\alpha^{\prime} \alpha y\right)$ respectively. The solution of the non-homogeneous system corresponding to $(\alpha y)$ can frequently be found in terms of these functions.

Theorem 2. The solution of the system

where

$$
\left\{\begin{aligned}
P y & =f(x) \\
{\left[A_{i} y\right]_{a}+\left[B_{i} y\right]_{b} } & =0, \quad i=1,2, \ldots, p
\end{aligned}\right.
$$

$$
f(x)=\sum_{n=1}^{\infty} a, \psi \psi_{n}(x)
$$
stated.

1 Bôcher, Annals of Mathematics, 13 (1911), 71-88. Not $K(\zeta, x)$ as sometimes

2 Math. Ann., 63 (1907), 433-476. p. 461.

${ }^{3}$ Cf. Schmidt, loc. cit., or Courant-Hilbert, Methoden der Mathematischen Physik, 1 (1924), 137. 
the series being uniformly convergent in $(a, b)$, is

$$
y=\sum_{n=1}^{\infty} \frac{a_{n}}{\lambda_{n}} \phi_{n}(x) \text {. }
$$

For the solution is known to be ${ }^{1}$

$$
y=\int_{a}^{b} K(x, \zeta) f(\zeta) d \zeta
$$

and the series for $f(x)$ can be integrated term by term.

Two operator systems $(\alpha)$, $(\beta)$ may be said to be permutable if $(\alpha \beta)$ is the same as $(\beta a)$. For this it is necessary but not sufficient that $P Q=Q P$. If $P, Q$ are any two operators satisfying this condition the systems

$\left\{\begin{array}{c}P \\ {\left[D^{s}\right]_{a}, \quad s=0,1, \ldots, p-1}\end{array} \quad\right.$ and $\quad\left\{\begin{array}{c}Q \\ {[D]_{a}, \quad s=0,1, \ldots, q-1}\end{array}\right.$

are permutable, both products being equivalent to the system

$$
\left\{\begin{array}{l}
P Q \\
{\left[D^{s}\right]_{a}, \quad s=0,1, \ldots, p+q-1 .}
\end{array}\right.
$$

$D$ denotes $d / d x$. Again, Theorem 1 shows that a system is permutable with any power of itself, so that permutable systems involving twopoint boundary conditions exist.

It may be added in conclusion that if $(\alpha),(\beta)$ are self-adjoint operator systems, $(\alpha \beta)$ is not self-adjoint unless $(\alpha),(\beta)$ are permutable. This corresponds to the fact that the product of two symmetrical matrices is not symmetrical unless the matrices commute.

${ }^{1}$ Ince, op. cit., 256. (ay) is assumed to be incompatible.

\section{ACKNOWLEDGMENT.}

The result given in $\$ 1$ of the writer's paper " A note on the correlation of classes," these Proceedings, 1 (1927), 47-48, is due to S. Banach, Fundamenta Mathematicae, 6 (1924), 236. 